\title{
Using Coplink to Analyze Criminal-Justice Data
}

The Coplink system applies a concept spacea statistics-based, algorithmic technique that identifies relationships between suspects, victims, and other pertinent data-to accelerate criminal investigations and enhance law enforcement efforts.

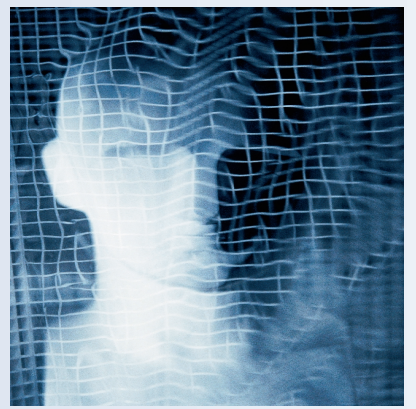

Roslin V. Hauck

Homa Atabakhsh

Pichai Ongvasith Harsh Gupta Hsinchun Chen University of Arizona

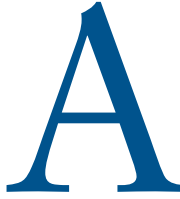

s information technologies and applications become more overwhelming and diverse, persistent information overload problems have become ever more urgent. ${ }^{1}$ Fallout from this trend has most affected government, specifically criminaljustice information systems. The explosive growth in the digital information maintained in the data repositories of federal, state, and local criminal-justice entities and the spiraling need for cross-agency access to that information have made utilizing it both increasingly urgent and increasingly difficult.

Incompatible content and information formats ${ }^{2}$ often create barriers to data access and utilization that make knowledge management a complex and daunting process. Nevertheless, numerous applications and approaches to knowledge management technologies have emerged, including aerospace engineering, ${ }^{2}$ virtual enterprising, ${ }^{3}$ joint ventures, ${ }^{4}$ and digital libraries. ${ }^{5}$

Several initiatives have been established to address the problems facing digital government's law enforcement sector. Five bureaus, including the National Institute of Justice, are working together through the Office of Justice Programs Integrated Justice Information Technology Initiative to develop ways to use wired-information technologies to improve the effectiveness and fairness of the justice system through better information sharing. NIJ's Office of Science and Technology developed the Agile program and other government initiatives (http://www.ojp.usdoj.gov) primarily to address interoperability issues.
These initiatives motivated a proposal to develop the Coplink project, which unites the technical expertise of the University of Arizona's Artificial Intelligence Lab with the Tucson Police Department's law enforcement domain knowledge. Coplink serves the community by bridging the gap between conducting research in cutting-edge technologies and solving real-world problems such as helping police officers fight crime.

\section{COPLINK IN TUCSON}

The Coplink concept space application, which began as a research project, has evolved into a realtime system being used in everyday police work. The Tucson Police Department had evaluated its information technology and identified several problems that stem from lack of information sharing, integration, and knowledge management. The TPD agreed to participate with UA's Artificial Intelligence Lab in research to investigate the potential of using state-of-the-art, near-term, and cost-effective database, Intranet, and multimedia technologies to make justice information database integration, management, and access more effective.

The Coplink project attacks several problems existing in many law enforcement agencies by developing a model integrated system that lets law officers access and share information with other agencies. Coplink has the additional goal of developing consistent, intuitive, and easy-to-use interfaces and applications that support specific and often complex law enforcement functions and tasks. Although the scope of this project includes a mul- 
tilevel development plan incorporating different information technologies, our research focused on improving criminal-intelligence analysis.

Crime analysts and detectives create knowledge from information daily by analyzing and generalizing current criminal records. The TPD's records consist of approximately 1.5 million criminal case reports that contain details from criminal events spanning the period from 1986 to 1999 . Although investigators can access the Records Management System to tie together information to help solve cases and crimes, they must manually search the RMS data for connections or existing relationships. Combining information to create knowledge is often hampered by voluminous information examination, which requires exorbitant time and effort on the investigator's part. That different investigators are more or less proficient at locating relevant information only compounds this problem.

Law enforcement agencies can use potent intelligence tools to alleviate information overload, reduce the time required to analyze criminal records, and advance the investigation of current cases. The Coplink knowledge management system can provide the functionality of intelligence analysis that current RMS systems lack. This system functions as a tool that serves the same purpose as current knowledge management practices while systematically and robustly giving crime analysts and investigators the power to explore the entire data set for possible relationships.

\section{CONCEPT SPACE}

Coplink's underlying structure is the concept space, or automatic thesaurus, a statistics-based, algorithmic technique used to identify relationships between objects of interest. ${ }^{6} \mathrm{~A}$ concept space consists of a network of terms and weighted associations that assist in concept-based information retrieval within an underlying information space.

In addition, co-occurrence analysis uses similarity and clustering functions ${ }^{6}$ to weight relationships between all possible concept pairs. The resulting network-like concept space holds all possible associations between objects, which means that the system retains and ranks every existing link between every pair of concepts. Analysts frequently use this technique to develop domain-specific knowledge structures for digital-library applications.

In Coplink, detailed case reports serve as the underlying space, while concepts derive from the meaningful terms that occur in each case. Concept space analysis easily identifies relevant terms and their degree of relationship to the search term. The system output includes relevant terms ranked in the order of their degree of association, thereby distinguishing the most relevant terms from inconsequential terms. From a crime investigation standpoint, concept space analysis can help investigators link known objects to other related objects that might contain useful information for further investigation—such as people and vehicles related to a given suspect.

Information related to a suspect can direct an investigation to expand in the right direction, but a case report that reveals relationships among data in one particular case might fail to capture those relationships from the entire database. In effect, investigators need to review all case reports related to a suspect, which can be a tedious task. The Coplink project introduced concept space analysis as an alternative investigation tool that captures the relationships between objects in the entire database.

\section{BUILDING A CONCEPT SPACE}

In general, building a domain-specific concept space (CS) involves three main steps. First, to locate the sources from which they will derive terms or concepts, developers must identify document collections in a specific subject domain. For the TPD, the case reports in the existing database provided this information. The researchers categorized each piece of information in the TPD's case reports database and stored it in well-organized structures.

Next, the terms must be filtered and indexed. A co-occurrence analysis captures the relationships among indexed terms. Developers then insert the resulting concept space into a database for easy manipulation with an appropriate algorithm. These last two steps were customized for the Coplink CS. After optimizing the code and tuning the database, building a Coplink CS takes approximately five hours, an acceptable period considering the TPD's requirements.

Theoretically, a concept space can contain any number of term types, such as person names, organizations, locations, crime types, and so forth. In practice, however, the size of the database, the time required to build a concept space, and query response time pose major constraints that limit the number of term types. To balance performance and comprehensiveness, a concept space should contain only meaningful types that users frequently search. TPD personnel assisted in identifying and creating a set of term types for the Coplink CS.

Term types in the CS concept space were divided
Concept space analysis easily identifies relevant terms and their degree of relationship to the search term. 
After identifying terms, we then computed the term frequency and document frequency for each term in a document, based on the methodology developed by Hsinchun Chen and Kevin Lynch. ${ }^{1}$ Term frequency, $t f$, represents the number of occurrences of term $j$ in document $i$. Document frequency, $d f$, represents the number of documents in a collection of $N$ documents in which term $j$ occurs.

We then computed the combined weight of term $j$ in document $i, d_{i j}$, based on the product of term frequency and inverse document frequency as follows:

$$
d_{i j}=t f_{i j} \times \log \left(\frac{N}{d f_{j}} \times w_{j}\right)
$$

where $N$ represents the total number of documents in a collection and $w_{j}$ represents the weight of words in descriptor $j$. In general, some term types are more descriptive and more important than others. Thus, they are assigned higher weights to ensure that the system always ranks relationships associated with these types reasonably. In the Coplink CS, we assign crime types comparatively higher weights.

We then performed term co-occurrence analysis based on the asymmetric cluster function.

$$
\begin{aligned}
W_{j k}= & \frac{\sum_{i=1}^{n} d_{i j k}}{\sum_{i=1}^{n} d_{i j}} \times \text { WeightingFactor }(k) \\
W_{k j}= & \frac{\sum_{i=1}^{n} d_{i k j}}{\sum_{i=1}^{n} d_{i k}} \times \text { WeightingFactor }(j)
\end{aligned}
$$

$W_{j k}$ indicates the similarity weights from term $j$ to term $k$, and $W_{k j}$ indicates the similarity weights from term $k$ to term $j$. We calculated the terms $d_{i j}$ and $d_{i k}$ based on the equation in the previous step. These terms represent the combined weight of both descriptors $j$ and $k$ in document $i$. However, they were computed slightly differently due to their different starting terms. We define them as follows:

$$
d_{i j k}=t f_{i j k} \times \log \left(\frac{N}{d f_{j k}} \times w_{j}\right)
$$

$$
d_{i k j}=t f_{i j k} \times \log \left(\frac{N}{d f_{j k}} \times w_{k}\right)
$$

where $t f_{i j k}$ represents the number of occurrences of both term $j$ and term $k$ in document $i$-we chose the smaller number of occurrences between the terms; $d f_{j k}$ represents the number of documents in a collection of $N$ documents in which terms $j$ and $k$ occur together.

To penalize general terms-those that appear in many places-in the co-occurrence analysis, we developed the following weighting scheme, which resembles the inverse document frequency function.

$$
\begin{aligned}
\text { WeightingFactor }(k)= & \frac{\log \frac{N}{d f_{k}}}{\log N} \\
\text { WeightingFactor }(j) & =\frac{\log \frac{N}{d f_{j}}}{\log N}
\end{aligned}
$$

Terms with a higher $d f_{k}$ or $d f_{j}$ value had a smaller weighting factor value, which caused the co-occurrence probability to become smaller. In effect, the algorithm pushes general terms down in the co-occurrence table.

We still need to conduct significant research into using concept space with our proposed noun phrasing and entity extraction techniques. In the preceding example, human analysts manually identified entity types from database fields. In addition, the Tucson Police Department does not yet capture freetext narratives.

Many law enforcement agencies have begun to incorporate content-rich narratives in their record management systems. These narratives will provide a fertile testbed for combining noun phrasing and concept space analysis for intelligence identification.

\section{Reference}

1. H. Chen and K.J. Lynch, "Automatic Construction of Networks of Concepts Characterizing Document Databases," IEEE Trans. Systems, Sept./Oct. 1992, pp. 885-902. into five main categories: Person, Organization, Location, Crime, and Vehicle. For the first four categories, only one piece of information such as a person's full name, street address, or crime type can function as a search term. For a vehicle, on the other hand, a single piece of information-such as color, make, or type-results in so many matches that using it as a search term would generate a flood of relevant terms. To avoid this problem, the Coplink CS combines two or more nonspecific vehicle terms into one composite term.

The index maintains the relationship between a term and the document in which it occurs. Cooccurrence analysis requires both an index and a 
reverse index. The index contains the links from term to document, while the reverse index contains the links from document to term. The "Applying Co-Occurrence Analysis to a Concept Space" sidebar describes the algorithm for creating these indexes in detail.

\section{GRAPHICAL USER INTERFACE}

The Coplink CS's graphical user interface allows entry of search terms from any of its four search forms: Person, Organization, Location, and Vehicle. For two or more search terms, an investigator can type each term in the relevant search form, then add them to the list through the Add button. The interface displays the list of search terms in the Display relationships between box shown in Figure 1. The concept space application can also display case reports with detailed information, including case number, team beat, crime type, and so forth.

The following scenario shows how the Coplink CS system and its interface work: A detective is investigating a robbery at a local convenience store. The crime's sole witness, the night store clerk, remembers only that the suspect drove away in a white pickup truck.

The detective needs to generate a lead from the type of crime and vehicle description. She selects the Vehicle search screen and enters "WHI" for the color white, "Pickup" for the vehicle's style, and "0304" as the universal crime report classification code for a convenience-store robbery. After adding the search terms to the relations box, the detective selects the Relationship button to enter the concept space shown in Figure 2. The user selects or deselects the types of relations the system returns, which allows the detective to choose only relevant categories and helps control information overload.

The system returns eight terms related to both a white pickup and the 0304 crime type. The detective now has a vehicle license plate number and knows that four people are somehow related to this type of crime and vehicle. She can always add any of the concept space terms to the search or remove one of the two keywords from it. As with the initial search screen, the panel in the lower left-hand corner lets the user control the amount of information the concept space returns. The detective now decides to access the Case details screen shown in Figure 3 to view any cases that underlie the relationships she has uncovered.

The Cases view displays actual case reports. For our scenario, the database contains only one applicable case. Using this view, the detective can exam-

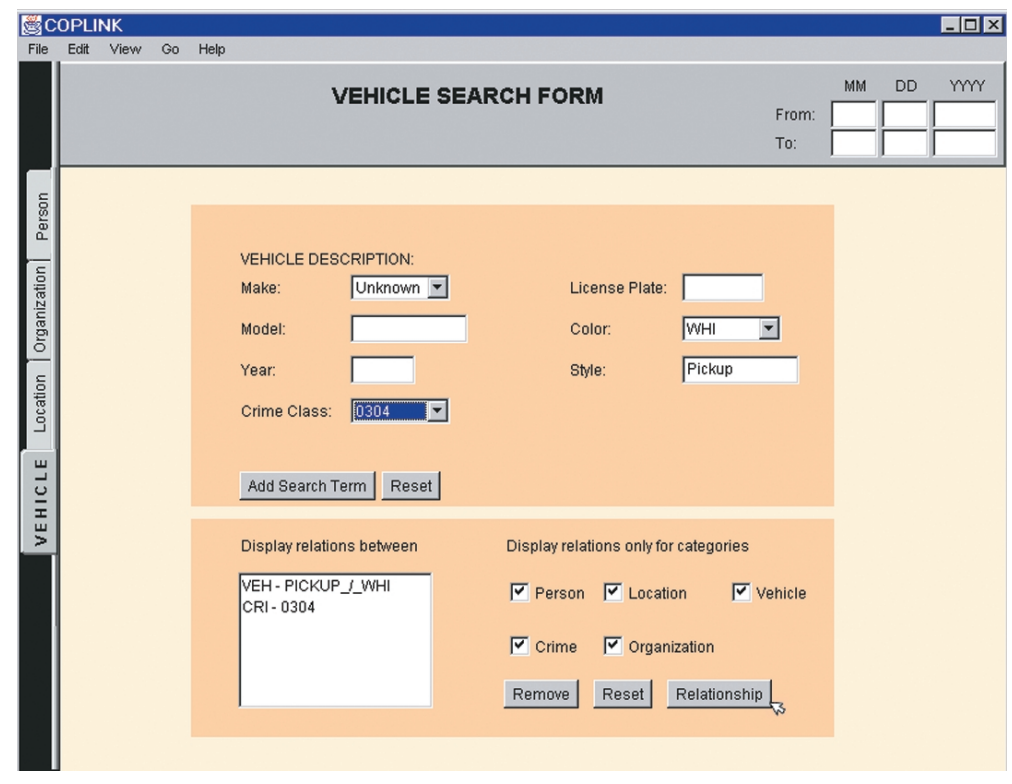

Figure 1. Concept space vehicle search screen. "Vehicle" is one of the information types users can enter as a search term. After adding the search terms to the relations box, the user selects the Relationship button to enter the concept space.

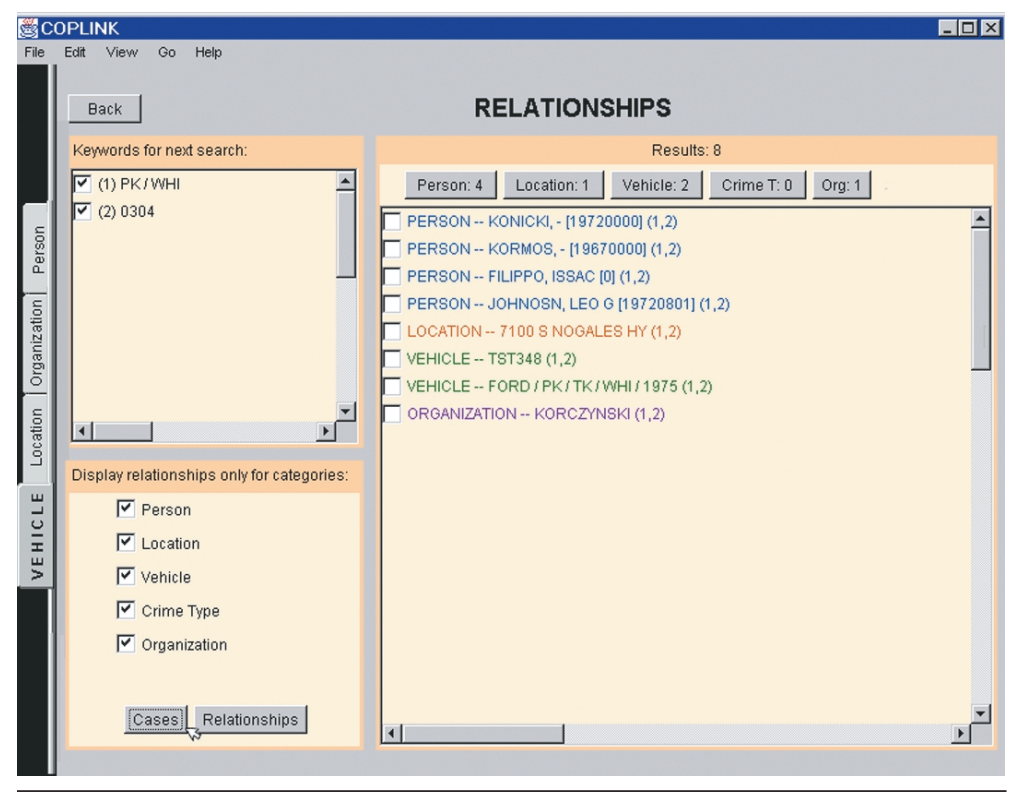

Figure 2. Relationships screen. The concept space can return elements for each of the five information object types: Person, Location, Vehicle, Crime type, and Organization. Here, the search has returned results for four persons, two vehicles, one location, and one organization.

ine the details of the prior incident, including the role and home address of each person involved. At any time, the detective can page back to review previous screens or modify the search keywords by selecting another type of search term or deselecting the current search terms.

\section{USER EVALUATIONS}

User evaluations helped to examine the effects of Coplink CS on law enforcement investigation 


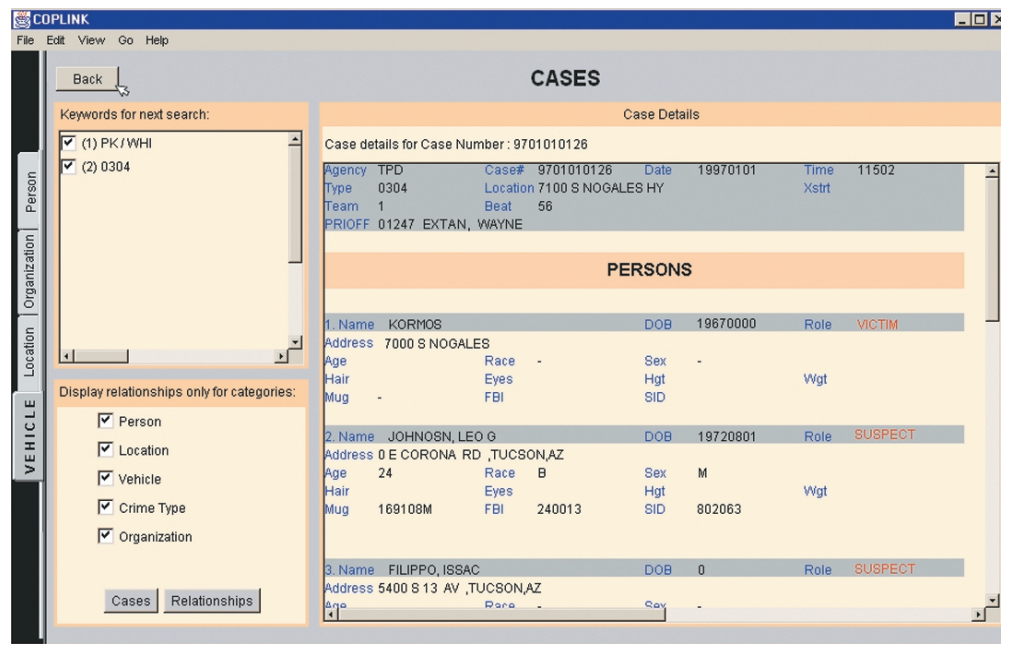

Figure 3. Case details screen. In this example, the detective can view the details of the one prior incident in the database, including the role and home address of each person involved. and work practices. ${ }^{78}$ The 12 crime analysts and detectives who participated in the longitudinal study completed journal entries on searches they had conducted using Coplink CS during a fourweek period. The researchers used documentation, structured interviews, and direct observation to collect data and evaluate the Coplink CS system's function and design. The evaluation of these information sources revealed three major areas in which Coplink CS provided support for intelligence analysis and knowledge management.

\section{Link analysis and summarization}

Participants indicated that the concept space application served as a powerful tool for acquiring information. They cited its value in helping determine the presence or absence of links between people, places, vehicles, and other object types in investigating a case. ${ }^{9}$

The impact of link analysis on investigative tasks is crucial to building cases. To begin working on a case, the investigating officer must have enough information to develop a lead. Too often, a case must be closed because investigators lack information or cannot use information that exists elsewhere in the RMS. Concept space manages the records system data so that investigators can use it to gain knowledge about the suspect.

Link analysis can directly link known information, indirectly link known information, or link unknown information. Study participants also reported that they used the concept space as a summary of the different information types related to a search term.

\section{Interface design}

In general, users reported that they found the Coplink CS Web-based interface engaging and easy to use. Officers noted that the graphical user inter- face and use of color to distinguish different object types provided a more intuitive tool than the textbased RMS system. Additionally, users can specify the type of information they need because the system returns results in either a concept space or case detail format.

Participants reported that the concept space application's data fields embody the basic information necessary for an investigation. In addition, the separation between different fields in the output effectively encourages easy comprehension of the information. A criminal investigation usually requires officers to make specific connections between details such as people, places, and vehicles to build a complete picture. Thus, the ability to aggregate information fields for a search provides a potent tool for problem solving and crime investigation.

\section{Operating efficiency}

Using Coplink CS in law enforcement offers one especially crucial benefit: speed. As one participant explained, it's difficult to identify a suspect after 48 to 72 hours have elapsed since the crime was committed. Beyond this time frame, a suspect can change his appearance to avoid identification or destroy evidence that may tie him to the crime. Witness and victim memory of the suspect's appearance also fades within this period. Thus, suspect identification should occur within 48 hours of the crime, a deadline that makes establishing useful links for identifying and locating the suspect vital. Several interview and journal comments indicated that using Coplink CS increased productivity by reducing the time spent per information search.

In journals and interview sessions, each participant reported the time it took to complete at least one particular search task using both RMS and Coplink CS. The data indicated that in a direct comparison of 15 searches, using Coplink CS required an average of 30 minutes less per search than did RMS. In addition, review of other qualitative data from participants' journals and interviews indicated that subjects perceived much quicker responses to queries from Coplink CS than from RMS, especially when the queries involved multiple search entries and query expansion.

Multiple search entries. The Coplink CS allows users to enter multiple search terms, a capability that the current RMS system lacks. With RMS, an officer must conduct several single searches, and then manually compare them. Consequently, using the RMS can take a few hours to accomplish what a concept space analysis can do within seconds.

Query expansion. Users can add any terms to their 
search that the concept space returns. The point-andclick action lets users add any number of terms to expand searches quickly and explore more searches in a shorter time. In addition, users can view concept spaces or documents on terms returned from previous searches without retyping the query.

\section{CURRENT USE}

Coplink CS has been successfully deployed at the Tucson Police Department, where crime analysts, officers, detectives, and sergeants from 16 departmental units use the technology voluntarily as part of their daily investigative routine. This cross-section comprises approximately 30 to 40 percent of TPD's investigative units and includes 90 percent of the crime analysts. Investigative units include adult and child sexual assault, aggravated assault, auto theft, elder abuse, community office, fraud, gangs, homicide, undercover, neighborhood crimes, night detectives, patrol, robbery, and warrants.

TPD's use of Coplink CS was closely monitored during a seven-week period, documenting statistics on frequency of use and following up with real case activities. During that period, Coplink CS completed 84 percent of 965 searches in less than three seconds.

In addition to documenting Coplink CS's efficiency, the study also confirmed its ease of use. Users who received training could become proficient with the system in minutes, while others who installed and learned the system on their own also found it easy to use.

Perhaps most importantly, Coplink CS proved effective in the investigation of real crimes, with improved case closure and solvability ratings. For example, one detective investigating a gang shooting explained how Coplink CS helped her investigation. When the detective accepted the case, she knew only the suspected shooter's moniker or alias. Unfortunately, several other gang members also used this moniker.

Using Coplink CS, the detective cross-referenced the moniker with the name of the victim, searching for associations, then generated a photo lineup using Coplink criminal mug shots. The witness identified the suspect from the assembled photos, leading to the apprehension and arrest of the suspected shooter. Using Coplink CS for the investigation and generating the photo lineup took the detective about five to 10 minutes. Without the concept space, the search could have taken days and required the assistance of several crime analysts.

In addition to the 100-plus detectives and crime analysts who are using Coplink at TPD, several large law enforcement agencies in California, Texas, and
Michigan are considering adopting the system for use in their investigative work.

\section{SECURITY AND PRIVACY}

Although all organizations must be concerned with the security and private exchange of information, this issue carries special weight for law enforcement agencies. Coplink incorporates several measures that help to ensure security and privacy.

First, users can access the system only through the Coplink node, which ensures that their queries never penetrate directly to the underlying database systems. This enhances security by letting agencies select the information they want to share while protecting other information, such as local personnel data and pending investigations. Second, using intranet and extranet functions- such as firewalls, IP address checks, user password authorizations, and database audit trails-helps in tracking system access and usage.

Coplink CS encrypts and compresses information using HTTPS/IP over a dedicated line or virtual private network. In the TPD, Coplink resides behind a firewall. Only workstations with certain IP addresses and the correct user password can access the application. In addition, the network maintains audit trails of all queries run on Coplink so that system administrators can monitor all user activities.

To protect information privacy and prevent porting sensitive information into the node, the host agency uses a policy-guided solution to determine sharing parameters. For example, although the gang unit captures data on potential gang members, some of this information may be classified as intelligence-sensitive tactical and strategic information on potential but not verified criminal activity that therefore cannot be released to the entire department. Following Arizona statutes, gang information included in Coplink must meet at least two of the seven Gang Membership Identification Criteria codes.

\section{FUTURE DIRECTIONS}

Because criminals are creatures of habit, law enforcement officers can be more effective if they have information about perpetrators' habits and close associations. ${ }^{10}$ The Coplink CS takes advantage of this characteristic by capturing connections between people, places, events, and vehicles, based on past crimes. The initial evaluation of this intelligence analysis application supports its potential for transforming law enforcement knowledge management practices.
To protect information privacy, the host agency uses

a policy-guided solution to determine sharing parameters. 


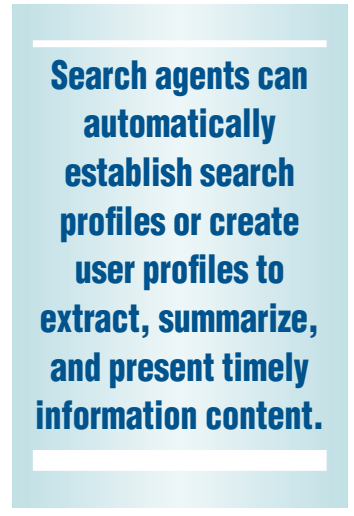

This particular project does not use entity extraction techniques because we drew the data from a structured database system. Yet many police records systems contain large collections of unstructured text and structured case reports. These textual sources often contain volumes of information that are not captured in the structured fields. A future research direction is to develop textual mining approaches that support knowledge retrieval from such sources. The development of linguistic-analysis and textual-mining techniques for performing fine-grained content analysis could help to make intelligent use of large textual collections in police databases.

Researchers are exploring various entity-extraction techniques. For example, the MENE system, ${ }^{11}$ developed at NYU, uses a rules-based approach in combination with a feature-importance component supported by maximum-entropy-probability calculation. Another approach, NetOwl, uses name recognition rules with a name database lookup. ${ }^{12}$ Although both of these systems have performed well on specific text types, generalizing them to different domains may be problematic given the rulebased nature of their operation. Yet with police report narratives, generalizability will be key to system performance. We are working with the Phoenix Police Department's collection of criminal narratives to develop and test a technique that uses a combination of noun phrasing, a finite-state automata system, and artificial neural networks to perform entity extraction.

Several Internet research projects have shown the power of a new agent-based search paradigm. In addition to supporting conventional searches, search agents can automatically establish search profiles or create user profiles to extract, summarize, and present timely information content. Such a proactive search agent would be well suited for use by investigative personnel in law enforcement agencies. We plan to develop a personalized law enforcement search agent that will support wide expansion in connectivity and information sharing between police agencies.

$\mathrm{R}$ esearchers in the University of Arizona's Artificial Intelligence Lab originally generated the concept space approach to facilitate semantic retrieval of information. Although Coplink's current incarnation demonstrates its potential as a tool for enhancing the effectiveness of law enforcement, new technologies will provide opportunities for making the system even more useful. As the computing industry continues to develop distributed solutions and analysis tools, a specific focus is to provide tools for use within the constraints of a wireless environment. We thus seek to develop and refine applications to support the expansion of distributed and mobile law enforcement networks and interjurisdictional information retrieval and to investigate and study network security issues.

Meanwhile, research into applying the concept space approach to other domains continues. Several user studies have shown that this system also improves searching and browsing in the engineering and biomedicine domains. In the biosciences, developers have applied the concept space technique to the Worm Community System and the FlyBase system. This approach has also been applied successfully in the Digital Library Initiative studies conducted on the INSPEC collection for computer science and engineering and for Internet searching. 5,7

\section{Acknowledgments}

This project was funded by grants from the National Institute of Justice, Office of Science and Technology (\#97-LB-VX-K023), and the National Science Foundation (\#9983304), with support from the Digital Equipment Corporation External Technology Grants Program, agreement \#US-1998004, which provided an equipment grant. We thank Joanne Martinez and Kristen Tolle for their feedback, and Jennifer Schroeder, Linda Ridgeway, and all the other personnel from the Tucson Police Department who participated in this project.

\section{References}

1. D.C. Blair and M.E. Maron, "An Evaluation of Retrieval Effectiveness for a Full-Text DocumentRetrieval System," Comm. ACM, vol. 28, no. 3, 1985, pp. 289-299.

2. P. Jones and J. Jordan, "Knowledge Orientations and Team Effectiveness," Int'l J. Technology Management, vol. 16, nos. 1-3, 1998, pp. 152-161.

3. Y.M. Chen, C.C. Liao, and B. Prasad, "A Systematic Approach of Virtual Enterprising Through Knowledge Management Techniques," Concurrent Engineering-Research and Applications, vol. 6, no. 3, 1998, pp. 225-244.

4. A.C. Inkpen and A. Dinur, "Knowledge Management Processes and Joint International Ventures," Organization Science, vol. 9, no. 4, 1998, pp. 454-468. 
5. H. Chen, “Artificial Intelligence Techniques for Emerging Information Systems Applications: Trailblazing Path to Semantic Interoperability," J. Am. Soc. Information Science, vol. 49, no. 7, 1998, pp. 579-581.

6. M. Lesk, Practical Digital Libraries, Morgan Kaufmann, Los Altos, Calif., 1997.

7. H. Chen and D.T. Ng, "An Algorithmic Approach to Concept Exploration in Large Knowledge Network (Automatic Thesaurus Consultation): Symbolic Branch-and-Bound vs. Connectionist Hopfield Net Activation," J. Am. Soc. Information Science, vol. 46, no. 5, 1995, pp. 348-369.

8. R.V. Hauck and H. Chen, "Coplink: A Case of Intelligent Analysis and Knowledge Management," Proc. 20th Ann. Int'l Conf. Information Systems, Assoc. Information Sys., Atlanta, 1999, pp. 15-28; http://ai. bpa.arizona.edu/go/datawarehousing/publications/ icispdf.pdf.

9. W.R. Harper and D.H. Harris, "The Application of Link Analysis to Police Intelligence," Human Factors, vol. 17, no. 2, 1975, pp. 157-164.

10. N. Joyce and J. Lewin, “ICAM: Chicago's Newest Crime-Fighting Tool,” Proc. Conf. Technology, Community Policing, National Law Enforcement and Corrections Technology Center, 1996, http://www. nlectc.org/txtfiles/confrpt.html.

11. A. Borthwick et al., "NYU: Description of the MENE Named Entity System as Used in MUC-7," Proc. 7th Message Understanding Conf., Science Applications Int'l,1998, http://www.itl.nist.gov/iaui/894.02/related _projects/muc/proceedings/muc_7_proceedings/ isoquest.pdf.

12. G.R. Krupka and K. Hausman, "Description of NetOwl Extractor System as Used in MUC-7," Proc. 7th Message Understanding Conf., Science Applications Int'l, 1998, http://www.itl.nist.gov/iaui/894.02/ related_projects/muc/proceedings/muc_7_proceedings/ nyu_english_named_entity.pdf.

Roslin V. Hauck is a doctoral candidate in the Department of Management Information Systems at the University of Arizona. Her research interests include technology adoption, organizational behavior, knowledge management, human-computer interaction, and usability. She received an MS in communication from the University of Arizona. Contact heratrrv@bpa.arizona.edu.

Homa Atabakhsh is principal research specialist at the University of Arizona MIS Department and is the Associate Director for the Coplink Center. She received a $\mathrm{PhD}$ in computer science from the University of Toulouse. Contact her at homa@bpa. arizona.edu.
Pichai Ongvasith is a database administrator in the Platform Technology group at J.D. Edwards. His research interests include database technology, Web applications, and enterprise resource planning systems. He received an MS in management information systems from the University of Arizona. Contact him atpichai_ongvasith@jdedwards.com.

Harsh Gupta is a database administrator at Microsoft in Redmond, Wash. His research interests include data migration issues and techniques for large data warehouses. He received an MS in management information systems from the University of Arizona. Contact him at gupta_harsh@hotmail. com.

Hsinchun Chen is McClelland Professor of Management Information Systems and head of the Artificial Intelligence Lab in the Department of Management Information Systems at the University of Arizona. His research interests include semantic retrieval, search algorithms, knowledge discovery, and collaborative computing. He received a $\mathrm{PhD}$ in information systems from New York University. Contact him at hchen@bpa. arizona.edu. 


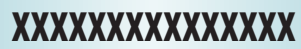
XXXXXXXXXXXXXXXXXX XXXXXXXXXXXXXXXXXX XXXXXXXXXXXXXXXXX $X X X X X X X X X X X X X X X X$ XXXXXXXXXXXXXXXXX XXXXXXXXXXXXXXXXXX XXXXXXXXXXXXXXXX XXXXXXXXXXXXXXXXXX 